\title{
Glutathione S-transferase polymorphisms in varicocele patients: a meta-analysis
}

\author{
B. Zhu', L. Yin ${ }^{2,3}$ and J.Y. Zhang ${ }^{1}$ \\ ${ }^{1}$ Biochemistry Department, Luohe Medical College, Luohe, Henan Province, China \\ ${ }^{2}$ Colllege of Life Science, Henan Normal University, Xinxiang, \\ Henan Province, China \\ ${ }^{3}$ Biochemistry Department, Luohe Medical College, Luohe, Henan Province, China
}

Corresponding author: B. Zhu

E-mail: baoan_zhu@126.com

Genet. Mol. Res. 14 (4): 18851-18858 (2015)

Received September 7, 2015

Accepted November 15, 2015

Published December 28, 2015

DOI http://dx.doi.org/10.4238/2015.December.28.34

\begin{abstract}
The glutathione S-transferase (GST) family represents a major group of detoxification and antioxidant enzymes. Studies have shown that high oxidative stress levels are associated with varicocele. The objective of this study was to assess the relationship between GSTM1 and GSTT1 null polymorphisms and varicocele using a study group of 497 varicocele patients and 476 control subjects. A systematic literature search (for articles published up to September 2014) utilizing Google Scholar and PubMed was conducted. The chi-square-based $Q$ test and $l^{2}$ index were used to evaluate data from retrieved studies. The possible publication bias was evaluated by Begg funnel plot and the Egger test. No statistically significant association was found between GSTM1 or GSTT1 null genotypes and varicocele in the overall data analysis. In a subgroup analysis, only the null GSTM1 genotype was observed at a significantly higher frequency in Caucasian varicocele patients. In the Chinese subgroup, no association
\end{abstract}


was established between the GSTM1 and GSTT1 null genotypes and this condition. More attention should be drawn to oxidative stress-related pathological manifestations for Caucasian varicocele patients.

Key words: GSTM1; GSTT1; Meta-analysis; Polymorphism; Varicocele

\section{INTRODUCTION}

Varicoceles (dilations of the pampiniform venous plexus) are found in approximately $15 \%$ of the general adult male population, a figure that increases to $35 \%$ for men presenting with primary infertility, and $81 \%$ for those with secondary infertility (Gorelick and Goldstein, 1993). The exact pathophysiology of varicocele remains unknown, but the most widely accepted concept is that it leads to elevated testicular temperature, which has a deleterious effect on spermatogenesis (Goldstein and Eid, 1989). Several studies have also suggested that this condition is associated with increased oxidative stress (Saleh et al., 2003; Allamaneni et al., 2004; Mancini et al., 2004), and reactive oxygen species (ROS) are found at significantly increased levels in varicocele patients compared to control groups (Hendin et al., 1999).

In testis tissues, GSTA, GSTM, GSTT, and GSTP, which belong to the glutathione S-transferase gene family, act as important protective factors against oxidative stress (Strange et al., 2001). The homozygous deletion (null genotype) of the GSTM1 or GSTT1 gene results in the total absence of corresponding enzyme activity. Varicocele patients' susceptibility to ROS may be due to one or more of these GST genetic polymorphisms. As GSTs are important for male reproduction and their malfunctioning is involved in impairment of spermatogenesis, deletion polymorphisms of GSTM1 or GSTT1 might be related to male infertility.

Only a small number of studies have focused on the association between GST genotypes and varicocele, and their results are contradictory. We thus carried out the current meta-analysis to assess the association between GSTM1 and GSTT1 gene polymorphisms and varicocele, in an attempt to evaluate possible pathophysiologies.

\section{METHODS}

\section{Data collection, extraction, and study design}

We conducted a systematic literature search (for articles published up to September 2014) of Google Scholar and PubMed databases using the following keywords: "GST-M1", "GST-T1", and "varicocele". The primary reports retrieved were filtered using the following inclusion criteria: 1) studies must consist of a case-control study of GSTM1 or GSTT1 polymorphisms and varicocele; and 2) must provide detailed genotype data. Non-case-control studies, reviews, meta-analyses, and duplicate data were excluded. The following information was independently extracted from eligible studies by two investigators ( $x x$ and $x x$ ), and tabulated: first author and year of publication, country, ethnicity, frequency of each genotype in case and control groups, and genotyping method. All disagreements were resolved by consensus between authors. Subgroup analyses were performed based on ethnicity (Caucasian and Asian) according to the studies involved. A detailed data collection flow chart is shown in Figure 1, and the characteristics of each included study are given in Table 1. 


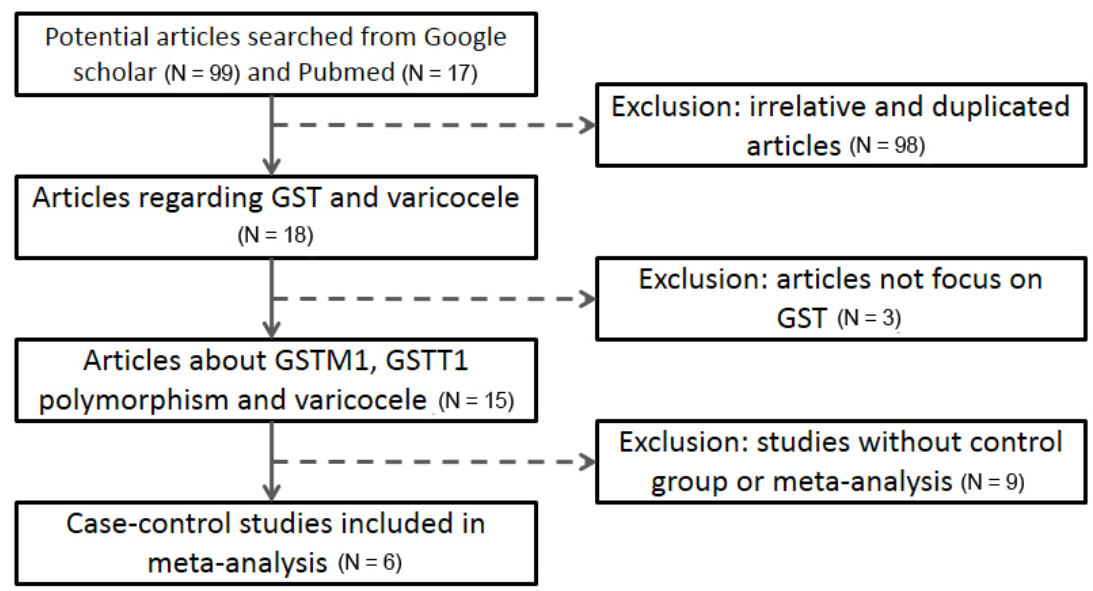

Figure 1. Flow chart describing the identification and retrieval of eligible studies. GST = glutathione S-transferase.

Table 1. Characteristics of each eligible study included in meta-analysis.

\begin{tabular}{|c|c|c|c|c|c|c|c|}
\hline \multirow[t]{2}{*}{ First author } & \multirow[t]{2}{*}{ Country } & \multirow[t]{2}{*}{ Ethnicity } & \multicolumn{2}{|c|}{ No. of patients for GSTM1 } & \multicolumn{2}{|c|}{ No. of patients for GSTT1 } & \multirow[t]{2}{*}{ Genotyping method } \\
\hline & & & Cases & Controls & Cases & Controls & \\
\hline Dehghani et al. (2014) & Iran & Caucasian & 46 & 48 & 46 & 48 & Multiplex PCR \\
\hline Tang et al. (2012) & China & Asian & 65 & 30 & 65 & 30 & Multiplex PCR \\
\hline Acar et al. (2012) & Turkey & Caucasian & 109 & 123 & 109 & 123 & Multiplex PCR \\
\hline Ichioka et al. (2009) & Japan & Asian & 72 & 101 & 72 & 101 & Multiplex PCR \\
\hline Wu et al. (2009) & China & Asian & - & - & 63 & 54 & Multiplex PCR \\
\hline Chen et al. (2002A) & China & Asian & 80 & 60 & - & - & Multiplex PCR \\
\hline Chen et al. (2002B) & China & Asian & 62 & 60 & - & - & Multiplex PCR \\
\hline
\end{tabular}

$\mathrm{PCR}=$ polymerase chain reaction

\section{Statistical methods}

Odds ratios (ORs) with $95 \%$ confidence intervals (Cls) were estimated for each study under the null vs present genotype genetic model to explore associations between GSTM1 and GSTT1 and varicocele. Each eligible study was weighted by sample size. Heterogeneity among studies was evaluated by the chi-square-based $Q$ test and $l^{2}$ index. When no heterogeneity $\left(I^{2}\right.$ $<50 \%$, $\mathrm{P}$ value $>0.5$ ) was observed, the fixed-effects model (Mantel-Haenszel method; Mantel and Haenszel, 1959) was applied for OR estimation. Otherwise, the random-effects model was used. Begg's funnel plot and the Egger test were used to evaluate possible publication bias. All analyses were performed using the Stata software (version 12; StataCorp, College Station, TX, USA).

\section{RESULTS}

\section{Subject characteristics}

Sixeligible case-control studies incorporating 497 cases and 476 controls were included 
in our meta-analysis (Chenet al., 2002; Ichioka et al., 2009; Wu et al., 2009; Acar et al., 2012; Tanget al., 2012; Dehghani et al., 2014). The characteristics of these studies are shown in Table 1. For analysis of GSTM1 polymorphism, the case group comprised 434 patients and the control group 422 individuals; for GSTT1, 355 patients were included, along with 356 healthy controls. All polymorphisms were assessed by multiplex polymerase chain reaction genotyping. Five studies were conducted in Asian populations, while two involved Caucasian subjects. Data regarding null, denoted as GSTM1(-) and GSTT1(-) hereafter, and present genotypes, denoted as GSTM1(+) and GSTT1(+) hereafter, are shown in Table 2.

\begin{tabular}{|c|c|c|c|c|c|c|c|c|}
\hline \multirow{2}{*}{ First author } & \multicolumn{2}{|c|}{ Cases } & \multicolumn{2}{|c|}{ Controls } & \multicolumn{2}{|c|}{ Cases } & \multicolumn{2}{|c|}{ Controls } \\
\hline & GSTM1 (-) & GSTM1 (+) & GSTM1 (-) & GSTM1 (+) & GSTT1 (-) & GSTT1 $(+)$ & GSTT1 (-) & GSTT1 (+) \\
\hline Dehghani & 28 & 18 & 20 & 28 & 22 & 24 & 24 & 24 \\
\hline Tang & 31 & 34 & 13 & 17 & 29 & 36 & 15 & 15 \\
\hline Acar & 50 & 59 & 46 & 77 & 24 & 85 & 28 & 95 \\
\hline Ichioka & 45 & 27 & 53 & 48 & 34 & 38 & 51 & 50 \\
\hline Wu & - & - & - & - & 32 & 31 & 23 & 31 \\
\hline Chen A & 35 & 45 & 27 & 33 & - & - & - & - \\
\hline Chen B & 26 & 36 & 27 & 33 & - & - & - & - \\
\hline
\end{tabular}

\section{Meta-analysis}

Heterogeneity tests of the overall datasets returned $l^{2}$ values of $0 \%$ for both genes, with $P$ values $>0.05$ (Table 3 ). Thus, no statistically significant heterogeneity was observed and we therefore used a fixed-effects model to assess associations within the data. In the overall analysis, no significant association was detected between genotype and varicocele for either GSTM1 (GSTM1(-) vs GSTM1(+); OR $=1.29,95 \% \mathrm{Cl}=0.98-1.70, \mathrm{P}=0.07$ ) or GSTT1 (GSTT1(-) vs GSTT1(+); OR $=0.97,95 \% \mathrm{Cl}=0.71-1.33, \mathrm{P}=0.87$; Figure $2 \mathrm{~A}$ and $\mathrm{B}$ ). Potential publication bias in the overall dataset was determined by generating Begg's funnel plots and applying Egger's linear regression test. The Begg's funnel plot for both GSTM1 and GSTT1 was symmetric (Figure 3), and the Egger's test $P$ values were both greater than 0.05 (Table 3).

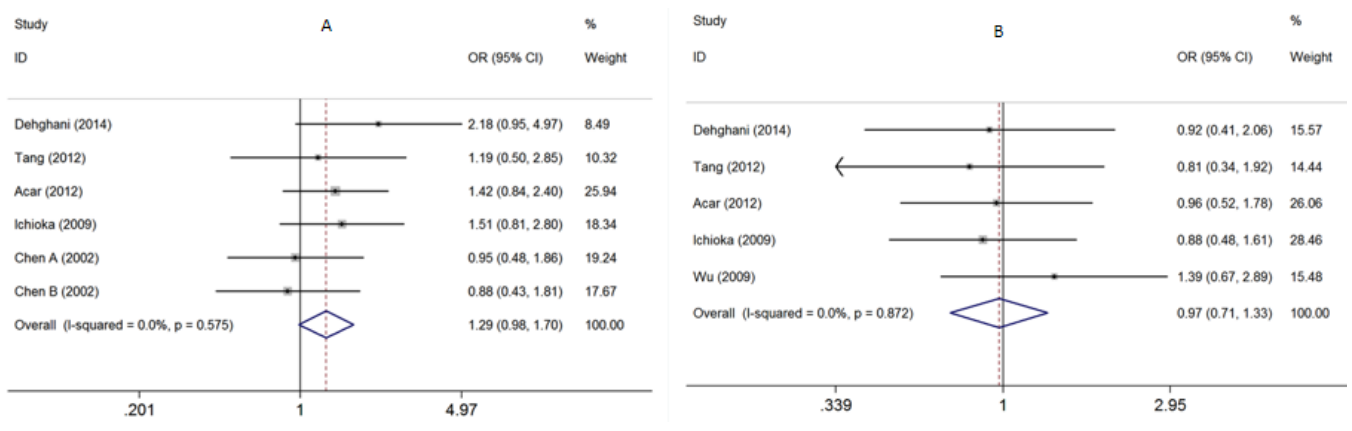

Figure 2. Results of the meta-analysis using the overall dataset. A. GSTM1 null vs GSTM1 present genotype. B. GSTT1 null vs GSTT1 present genotype. GST = glutathione S-transferase; OR = odds ratio; $\mathrm{Cl}=$ confidence interval. 

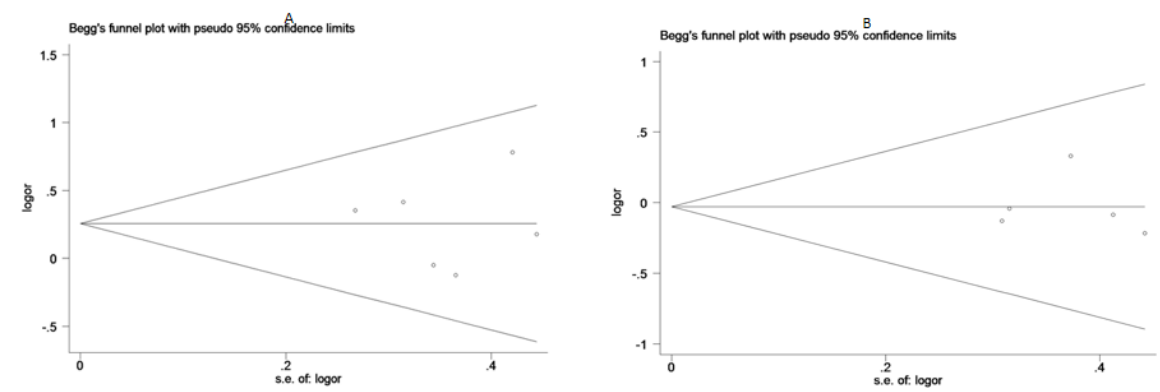

Figure 3. Begg's funnel plots relating to the association between GSTM1 and GSTT1 null polymorphisms and varicocele using the overall dataset. A. GSTM1 null vs GSTM1 present genotype. B. GSTT1 null vs GSTT1 present genotype. GST = glutathione S-transferase; SE = standard error; OR = odds ratio.

\section{Table 3. Results of the GSTM1 and GSTT1 meta-analysis.}

\begin{tabular}{|c|c|c|c|c|c|c|c|c|c|}
\hline \multirow[t]{2}{*}{ Analysis model } & \multirow[t]{2}{*}{ Analysis method } & \multicolumn{2}{|c|}{ Heterogeneity } & \multicolumn{4}{|c|}{ OR } & \multicolumn{2}{|c|}{ Publication bias } \\
\hline & & $1^{2}(\%)$ & $\mathrm{P}$ value $^{\#}$ & Overall & Lower & Upper & $P$ value $^{*}$ & Begg & Egger \\
\hline GSTM1(-) vs GSTM1(+) & Fixed & 0.0 & 0.575 & 1.29 & 0.98 & 1.70 & 0.07 & 1.00 & 0.96 \\
\hline GSTT1(-) vs GSTT1(+) & Fixed & 0.0 & 0.87 & 0.97 & 0.71 & 1.33 & 0.87 & 0.81 & 0.99 \\
\hline
\end{tabular}

$\mathrm{OR}=$ odds ratio. ${ }^{\mathrm{P} P}$ value from the heterogeneity test. ${ }^{*} \mathrm{P}$ value from the $\mathrm{OR}$ test.

Heterogeneity tests of the subgroup datasets gave similar results to those above, with ${ }^{2}$ values of $0 \%$ and $P$ values $>0.05$ for both genes, indicating no statistically significant heterogeneity in either Asian or Caucasian subsets (Table 4). Therefore, the fixed-effects model was applied to estimate ORs. Forest plots of the two subgroup analyses are shown in Figure 4. We found that the GSTM1(-) genotype was statistically strongly associated with varicocele in the Caucasian dataset $(\mathrm{OR}=1.61$, $95 \% \mathrm{Cl}=1.02-2.50, \mathrm{P}=0.03$; Figure $4 \mathrm{~A}$ ), while in the Asian subgroup, no significant association was detected $(\mathrm{OR}=1.13,95 \% \mathrm{Cl}=0.79-1.60, \mathrm{P}=0.54$; Figure 4B). However, analysis of the GSTT1 gene revealed no significant relationship in either the Caucasian $(\mathrm{OR}=0.94,95 \% \mathrm{Cl}=0.58-1.54, \mathrm{P}=0.81)$ or Asian datasets $(\mathrm{OR}=1.00,95 \% \mathrm{Cl}=0.66-1.50, \mathrm{P}=0.98$; Figure $4 \mathrm{C}$ and $\mathrm{D})$. Given the relatively small sample size involved, publication bias was not assessed for the subgroup analysis.
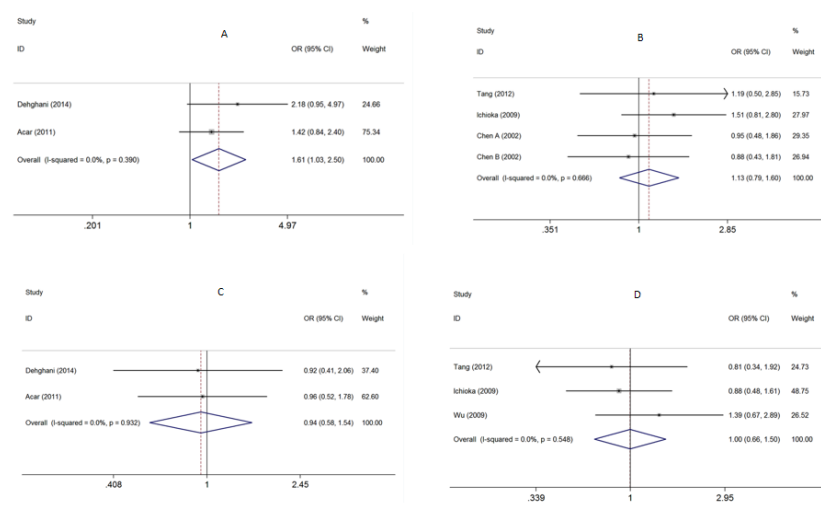

Figure 4. Forest plots from the subgroup analysis of A. GSTM1 in the Caucasian population, B. GSTM1 in the Asian population, C. GSTT1 in the Caucasian population, and D. GSTT1 in the Asian population. GST = glutathione S-transferase. 
Table 4. Subgroup meta-analysis for GSTM1 and GSTT1 based on ethnicity.

\begin{tabular}{|c|c|c|c|c|c|c|c|}
\hline \multirow[t]{2}{*}{ Analysis model } & \multirow[t]{2}{*}{ Analysis method } & \multicolumn{2}{|c|}{ Heterogeneity } & \multicolumn{4}{|c|}{ OR } \\
\hline & & $1^{2}(\%)$ & $\mathrm{P}$ value ${ }^{\#}$ & Overall & Lower & Upper & $P$ value* \\
\hline \multicolumn{8}{|l|}{ Caucasian } \\
\hline GSTM1(-) vs GSTM1(+) & Fixed & 0.0 & 0.39 & 1.61 & 1.03 & 2.50 & 0.03 \\
\hline GSTT1(-) vs GSTT1(+) & Fixed & 0.0 & 0.93 & 0.94 & 0.58 & 1.54 & 0.81 \\
\hline \multicolumn{8}{|l|}{ Asian } \\
\hline GSTM1(-) vs GSTM1(+) & Fixed & 0.0 & 0.67 & 1.13 & 0.79 & 1.60 & 0.54 \\
\hline GSTT1(-) vs GSTT1(+) & Fixed & 0.0 & 0.55 & 1.00 & 0.66 & 1.50 & 0.98 \\
\hline
\end{tabular}

$\mathrm{OR}=$ odds ratio. ${ }^{\# P}$ value from the heterogeneity test. ${ }^{*} \mathrm{P}$ value from the $\mathrm{OR}$ test.

\section{DISCUSSION}

The glutathione S-transferases are a family of isoenzymes that play important roles in protection against oxidative stress. Under aerobic conditions, human spermatozoa generate ROS (Holland et al., 1982) as a normal physiological process (de Lamirande and Gagnon, 1993; Aitken and Fisher, 1994). However, in healthy men's seminal plasma, excessive ROS are neutralized by antioxidants. When the balance between ROS production and antioxidant capacity is shifted, e.g. in pathological conditions where GST activity is reduced, surplus ROS might lead to sperm malfunction (Aitken and Clarkson, 1987; Alvarez et al., 1987; Gopalakrishnan and Shaha, 1998; Aydemir et al., 2007) and a high rate of DNA damage (Lopes et al., 1998).

Here, we presented an up-to-datemeta-analysis including 497 cases and 476 controls, investigating the role of GSTM1 and GSTT1 null polymorphisms in varicocele patients, in an attempt to explore possible pathophysiologies of this disease. Our results showed that in an overall population analysis, GSTM1 and GSTT1 null polymorphisms were not observed more frequently in varicocele patients than in control subjects. Similarly, Chen et al. (2002) also detected no difference between control and varicocele patient groups regarding the GSTM1 null genotype. Interestingly, only in the Caucasian subgroup analysis did we find a significantly higher frequency of the GSTM1 null genotype amongst varicocele patients, with an OR of $1.61(P=0.03)$. In contrast, no such association was discerned in the Asian subgroup. We failed to detect any statistically significant correlation concerning the GSTT1 null genotype in either Caucasian or Asian populations. As the sperm of varicocele patients with GSTM1 null genotypes are more vulnerable to oxidative damage (Chen et al., 2002), more attention should be paid to oxidative stress-related pathological manifestations for varicocele sufferers carrying such a null polymorphism. Although the impact of varicocele on male fertility remains unknown (Baazeem et al., 2011), reduced detoxification capacity during oxidative stress seems likely to be a contributory factor for those patients in Caucasian populations with a GSTM1 null genotype.

It should be noted that there are several limitations to this study. Firstly, only a small number of investigations have focused on the association between GSTM1 and GSTT1 polymorphisms and varicocele. Although we included a comprehensive, up-to-date list of publications, it would be preferable to incorporate further data for a more extensive meta-analysis, particularly for ethnicitybased subgroup tests. In addition, other members of the GST family might compensate for the loss of GSTM1 and GSTT1 activity. Due to the limited eligible data, only null genotypes of GSTM1 and GSTT1 were assessed in the current meta-analysis. Future studies should include other genetic polymorphisms of varicocele patients.

In conclusion, we investigated GSTM1 and GSTT1 polymorphisms in varicocele patients 
and found that only amongst those from Caucasian populations was the GSTM1 null genotype observed at a significantly higher frequency. The performance of epidemiological studies is strongly recommended to validate the role of the GSTM1 gene in male infertility in Caucasian populations.

\section{Conflicts of interest}

The authors declare no conflict of interest.

\section{ACKNOWLEDGMENTS}

Research supported by funding from Natural Science Foundation of China (\#31201093), and from the National Basic Research "973" Pre-Research Program of China (\#2012CB722304).

\section{REFERENCES}

Acar H, Kilinc M, Guven S and Inan Z (2012). Glutathione S-transferase M1 and T1 polymorphisms in Turkish patients with varicocele. Andrologia 44: 34-37.

Aitken J and Fisher H (1994). Reactive oxygen species generation and human spermatozoa: the balance of benefit and risk. Bioessays 16: 259-267.

Aitken RJ and Clarkson JS (1987). Cellular basis of defective sperm function and its association with the genesis of reactive oxygen species by human spermatozoa. J. Reprod. Fertil. 81: 459-469.

Allamaneni SS, Naughton CK, Sharma RK, Thomas AJ Jr, et al. (2004). Increased seminal reactive oxygen species levels in patients with varicoceles correlate with varicocele grade but not with testis size. Fertil. Steril. 82: 1684-1686.

Alvarez JG, Touchstone JC, Blasco L and Storey BT (1987). Spontaneous lipid peroxidation and production of hydrogen peroxide and superoxide in human spermatozoa. Superoxide dismutase as major enzyme protectant against oxygen toxicity. J. Androl. 8: 338-348.

Aydemir B, Onaran I, Kiziler AR, Alici B, et al. (2007). Increased oxidative damage of sperm and seminal plasma in men with idiopathic infertility is higher in patients with glutathione S-transferase Mu-1 null genotype. Asian J. Androl. 9: 108-115.

Baazeem A, Belzile E, Ciampi A, Dohle G, et al. (2011). Varicocele and male factor infertility treatment: a new meta-analysis and review of the role of varicocele repair. Eur. Urol. 60: 796-808.

Chen SS, Chang LS, Chen HW and Wei YH (2002). Polymorphisms of glutathione S-transferase M1 and male infertility in Taiwanese patients with varicocele. Hum. Reprod. 17: 718-725.

de Lamirande E and Gagnon C (1993). Human sperm hyperactivation in whole semen and its association with low superoxide scavenging capacity in seminal plasma. Fertil. Steril. 59: 1291-1295.

Dehghani M, Haghiralsadat F, Azimzadeh M, Vahidi S, et al. (2014). Association of glutathione S-transferase (GSTT1 and GSTM1) polymorphism with varicocele: an Iranian case-control study. Int. J. Biosci. 4: 146-153.

Goldstein M and Eid JF (1989). Elevation of intratesticular and scrotal skin surface temperature in men with varicocele. J. Urol. 142: $743-745$

Gopalakrishnan B and Shaha C (1998). Inhibition of sperm glutathione S-transferase leads to functional impairment due to membrane damage. FEBS Lett. 422: 296-300.

Gorelick JI and Goldstein M (1993). Loss of fertility in men with varicocele. Fertil. Steril. 59: 613-616.

Hendin BN, Kolettis PN, Sharma RK, Thomas AJ Jr, et al. (1999). Varicocele is associated with elevated spermatozoal reactive oxygen species production and diminished seminal plasma antioxidant capacity. J. Urol. 161: 1831-1834.

Holland MK, Alvarez JG and Storey BT (1982). Production of superoxide and activity of superoxide dismutase in rabbit epididymal spermatozoa. Biol. Reprod. 27: 1109-1118.

Ichioka K, Nagahama K, Okubo K, Soda T, et al. (2009). Genetic polymorphisms in glutathione S-transferase T1 affect the surgical outcome of varicocelectomies in infertile patients. Asian J. Androl. 11: 333-341.

Lopes S, Jurisicova A, Sun JG and Casper RF (1998). Reactive oxygen species: potential cause for DNA fragmentation in human spermatozoa. Hum. Reprod. 13: 896-900.

Mancini A, Meucci E, Milardi D, Giacchi E, et al. (2004). Seminal antioxidant capacity in pre- and postoperative varicocele. J. Androl. 25: 44-49.

Mantel N and Haenszel W (1959). Statistical aspects of the analysis of data from retrospective studies of disease. J. Natl. 
Cancer Inst. 22: 719-748.

Saleh RA, Agarwal A, Sharma RK, Said TM, et al. (2003). Evaluation of nuclear DNA damage in spermatozoa from infertile men with varicocele. Fertil. Steril. 80: 1431-1436.

Strange RC, Spiteri MA, Ramachandran S and Fryer AA (2001). Glutathione-S-transferase family of enzymes. Mutat. Res. 482: 21-26.

Tang K, Xue W, Xing Y, Xu S, et al. (2012). Genetic polymorphisms of glutathione S-transferase M1, T1, and P1, and the assessment of oxidative damage in infertile men with varicoceles from northwestern China. J. Androl. 33: 257-263.

Wu Q, Xing J, Xue W, Sun J, et al. (2009). Influence of polymorphism of glutathione S-transferase T1 on Chinese infertile patients with varicocele. Fertil. Steril. 91: 960-962. 